\title{
HiCrome Bacillus agar for presumptive identification of Bacillus and related species isolated from honey samples
}

\author{
Adriana M. Alippi*, Eliana Abrahamovich \\ CIDEFI - Facultad de Ciencias Agrarias y Forestales, Universidad Nacional de La Plata, calles 60 y 119 S/N, 1900 La Plata, Buenos Aires, Argentina
}

\section{A R T I C L E I N F O}

\section{Keywords:}

Aerobic spore-forming bacteria

Chromogenic media

Paenibacillus

Brevibacillus

Lysinibacillus

\begin{abstract}
A B S T R A C T
This study aimed to evaluate the performance of Hicrome Bacillus ${ }^{\mathrm{TM}}$ agar for isolation and rapid identification of the aerobic spore-forming bacteria most frequently found in honey samples. A collection of 197 bacterial isolates of Bacillus, Brevibacillus, Lysinibacillus, Paenibacillus, and Rummeliibacillus belonging to different species that have been reported in honey were screened for their abilities to grow and for their colony colors and medium appearance in HiCrome Bacillus agar. Also, 21 strains from culture collections were used for comparison and quality controls. A flowchart utilizing a combination of colony and media characteristics in the chromogenic medium and a set of simple biochemical and morphological tests were elaborated for quick presumptive identification. A procedure for direct isolation from honey samples was developed. In conclusion, HiCrome Bacillus agar in combination with simple microbiological tests was highly useful for rapid and reliable identification of most Bacillus, Brevibacillus, Lysinibacillus and Paenibacillus species commonly found in honey samples facilitating isolation from polymicrobial honey.
\end{abstract}

\section{Introduction}

Honey is a complex substance made up by gathering nectar and sweet deposits from plants and trees, modified and stored in honeycombs by various species of honey bees (Apis spp.).

Honey quality is influenced by microorganisms, mainly yeasts, and spore-forming bacteria; besides its use as a natural sweetener, honey is often used as a food ingredient, and its microbial load, mainly sporeforming bacteria, may be transferred to complex matrices (Sinacori et al., 2014). Aerobic spore-forming bacteria of the genus Bacillus and relatives are within the most common contaminants introduced in honey (Snowdon and Cliver, 1996) from digestive tracts of larvae and adult bees, brood combs, dust, air, earth, pollen, and nectar (Gilliam, 1979; Jeyaprakash et al., 2003; Piccini et al., 2004). Several species of these bacteria have been reported in honey on a regular basis, i.e. $\mathrm{Ba}$ cillus amyloliquefaciens, Bacillus cereus group, Bacillus circulans, Bacillus coagulans, Bacillus flexus, Bacillus licheniformis, Bacillus megaterium, Bacillus pumilus, Bacillus simplex, Bacillus subtilis, Brevibacillus brevis, Brevibacillus laterosporus, Lysinibacillus sphaericus, Paenibacillus alvei, Paenibacillus larvae and Paenibacillus polymyxa (Alippi, 1995; Alippi et al., 2004; Iurlina and Fritz, 2005, Silva et al., 2017, Sinacori et al., 2014; Snowdon and Cliver, 1996; Wen et al., 2017). According to their geographical source and botanical origin, honey showed variations in colony counts and microbial composition. Also, in the same honey sample, different species of spore-forming bacilli are frequently present ranging from the detection of a single colony to complete overgrowth of the plates (Alippi, 1995; Alippi et al., 2004) when using conventional culture media.

The use of traditional versus improved media formulation containing chromogenic substrates is a current topic in the field of microbiology. The focus behind such developments is to produce media that would make the detection and identification of microorganisms more rapid and reliable. Chromogenic substrates together with a specified selectivity of the medium are the simple principle behind chromogenic media (Perry and Freydiere, 2006). The target organisms are characterized by enzyme systems that metabolize the substrates to release the chromogen. The chromogen can then be visually detected by direct observation of a distinct color change in the medium. Direct confirmation of the target organism without further testing is sometimes possible (Siegrist, 2016).

HiCrome Bacillus agar was developed for Bacillus cereus isolation and enumeration from different types of food. The chromogenic mixture is cleaved by the enzyme $\beta$-glucosidase produced by the species of this group resulting in the formation of blue colonies. If selective isolation of $B$. cereus sensu lato is required, the antibiotic polymyxin B is added (Sigma-Aldrich, 2013). Also, the medium contains a peptic digest

\footnotetext{
* Corresponding author.

E-mail address: alippi@biol.unlp.edu.ar (A.M. Alippi).
} 
of animal tissues and meat extract, which provide nitrogenous compounds. Mannitol serves as the fermentable carbohydrate that can be detected by the $\mathrm{pH}$ indicator phenol red, so those species that utilized mannitol acidified the substrate, and phenol red turned to yellow. HiCrome Bacillus agar and other chromogenic media for identification of isolates within the Bacillus cereus group were used as an alternative to the conventional standard plating media (Fricker et al., 2008; Juergensmeyer et al., 2006; Nemecková et al., 2011; Tewari et al., 2013). Nevertheless, there is a lack of information about the usefulness of chromogenic media for detection and enumeration of other Bacillus species outside the Bacillus cereus group.

This study aimed to evaluate the performance of HiCrome Bacillus ${ }^{\mathrm{TM}}$ agar, recently renamed as Bacillus ChromoSelect agar (Sigma-Aldrich), for identification of the aerobic spore-forming bacteria most frequently found in honey samples in combination with simple microbiological tests.

\section{Materials and methods}

\subsection{Culture media}

HiCrome Bacillus agar ${ }^{\mathrm{TM}}$ (Fluka, Sigma-Aldrich) was prepared according to the manufacturer specifications with minor modifications and dispensed in screw-capped flasks. Briefly, flasks were boiled with flowing steam in an unpressurized autoclave $\left(100^{\circ} \mathrm{C}\right)$ during $45 \mathrm{~min}$. After boiling, the medium was homogenized under continuous stirring and cooled to $45^{\circ} \mathrm{C}$. Polymyxin B was not added to improve the recovery of Bacillus species beyond the B. cereus group. Then, $20 \mathrm{ml}$ was dispensed in sterile Petri plates $(90 \mathrm{~mm})$, allowed to solidified and stored at $4^{\circ} \mathrm{C}$ in the dark and used within a week.

\subsection{Bacterial strains}

A collection of 197 bacterial isolates of Bacillus, Brevibacillus, Lysinibacillus, Paenibacillus, and Rummeliibacillus belonging to different species that have been reported in honey were screened for their abilities to grow and for their colony colors and medium appearance in HiCrome Bacillus agar. The collection includes 167 isolates from honey samples and nine isolates from honeybee larvae. Also, 21 strains from Culture Collections were used for comparison and quality control purposes. Further details are provided in Supplementary Table 1 (Data in Brief). Bacteria were maintained as stock cultures at $-80^{\circ} \mathrm{C}$ in the correspondent broth medium, MYPGP (Dingman and Stahly, 1983) or BHI (Merck Química, Argentina) plus 20\% glycerol (v/v). For shortterm storage, the strains were kept at $4{ }^{\circ} \mathrm{C}$ in MYPGP or BHI semi-solid medium according to the species tested.

\subsection{Inoculation and color estimation in HiCrome Bacillus medium}

The inoculum was prepared as suspensions in $5 \mathrm{ml}$ of sterile distilled water from a 24-h agar plate of each bacterial isolate and control strains from Culture Collections and adjusted to a concentration of $0.5 \mathrm{Mac}$ Farland (equivalent to about $1 \times 10^{6}$ cells $/ \mathrm{ml}$ ). Five $\mu \mathrm{l}$ aliquots of each suspension were diluted in $50 \mu \mathrm{l}$ sterile distilled water and vortex mixed. Five $\mu$ l of each mixture was inoculated onto HiCrome Bacillus agar plates using a sterile Drigalsky spatula. Plates were prepared in duplicate and incubated at $30^{\circ}$ and $37^{\circ} \mathrm{C}$, respectively.

All the isolates and controls were compared with a Pantone ${ }^{\circledR}$ international printing color chart (http://www.cal-print.com/ InkColorChart.htm) determining colony appearance and color and any substrate color change at 24 and $48 \mathrm{~h}$ of incubation.

\subsection{Identification of bacterial strains}

All isolates were tested by colony morphology and microscopic examination of bacterial smears for the presence of unstained globules in the cytoplasm and size and location of spores according to standard protocols (Gordon et al., 1973; Parry et al., 1983). Bacterial cultures were also tested by selected test, i.e. catalase reaction, anaerobic growth, nitrate reduction, Voges-Proskauer reaction (VP), indol and urease production, mannitol, L-arabinose, and citrate utilization, starch and gelatin hydrolysis, decomposition of tyrosine, growth in $7 \%$ and $10 \%$ of $\mathrm{NaCl}$ and at different temperatures (30-37-50 and $55{ }^{\circ} \mathrm{C}$ ) according to standard protocols (Gordon et al., 1973; Parry et al., 1983; Priest et al., 1988). Any questionable results were confirmed by using API 20E and API 50CH strips plus API 50CHB medium (bioMérieux, France) according to the manufacturer's instructions. The interpretation of the results was made by using the identification software apiweb (bioMérieux, www.biomerieux.com).

\subsection{Sequence analysis}

Selected strains $(n=56)$ isolated from honey or honeybee larvae were further identified by sequencing the 16S rRNA. Universal eubacterial primers used for $16 \mathrm{~S}$ rRNA sequence analysis were $27 \mathrm{f} \mathrm{(}$ 5'-AGAGTTTGATCMTGGCTCAG-3') and 1492r (5'-TACGGYTACCTTGTTACGACTT-3') (Yu et al., 2013). PCR products of approximately $1400 \mathrm{bp}$ were purified by ethanol precipitation (Applied Biosystems, 2009) and sequenced by the dideoxy termination method by the commercial services of Macrogen Inc. (Seoul, Korea) or Unidad de Genómica, Instituto de Biotecnología, CICVYA - INTA (Hurlingham, Argentina). Sequence assembly and contig editing were performed by using CodonCode Aligner software (Codon Code Corporation, MA, USA). The partial sequences obtained were subjected to a Blast-N (http://www. ncbi.nlm.nih.gov) search to identify sequences with the highest similarity by comparison only with sequences obtained from cultures deposited in Culture Collections according to the criteria of Logan et al. (2009). Also, the obtained sequences were analyzed by EZBioCloud (http://www.ezbiocloud.n) by comparison with those from type cultures (Yoon et al., 2017).

Partial 16S rRNA sequences were aligned using Muscle (Edgar, 2004), together with those from type cultures of aerobic spore-formers reported in honey and other apiarian sources (Alippi, 1995; Alippi et al., 2004; Evans and Armstrong, 2006; Gilliam, 1979; Iurlina and Fritz, 2005, Piccini et al., 2004; Sinacori et al., 2014; Snowdon and Cliver, 1996; Wen et al., 2017). The analysis involved 93 nucleotide sequences comprising 56 sequences of Bacillus, Paenibacillus, Brevibacillus, Lysinibacillus, and Rummeliibacillus obtained in this study and 36 sequences obtained from GenBank. Sequences of type cultures used for comparisons are listed in Supplementary Table 2 (Data in Brief). A sequence of Micrococcus luteus [AJ536198.1] was used as an outgroup. Phylogenetic analysis was performed using both Neighbor-Joining (NJ) and Maximum likelihood (ML) methods available in Mega X program (Kumar et al., 2018). A bootstrap resampling analysis (Felsenstein, 1985), employing 1000 replicates, was used to assess the reliability of the phylogenetic trees (Nei and Kumar, 2000).

\subsection{Ecometric technique}

The Ecometric technique was used for comparative evaluation of HiCrome Bacillus agar and two control media, BHI or MYPGP according to the species tested. Overnight cultures growing on BHI or MYPGP agar were adjusted to $0.5 \mathrm{McF}$ arland in sterile distilled water. One loop of $10 \mu \mathrm{l}$ of each suspension was sequentially diluted from streak to streak onto each medium by inoculating 21 streaks ( 5 per quadrant and 1 in the center). Growth on the plates was recorded as a score. Readings were presented as absolute growth indices with possible values of 0 to 5 , where 0 is absence of growth in any streak and 5 was the maximum score obtained when all of the streaks in the four quadrants and also the last streak showed visible bacterial growth (Aguilera-Arreola et al., 2012; Kornacki et al., 2003). Twenty-eight bacterial strains with different colony types (listed in Supplementary Table 3 (Data in Brief) 
were used for the evaluation. Plates were inoculated and incubated in duplicate for $24-48 \mathrm{~h}$ at $37^{\circ} \mathrm{C}$. Scores for HiCrome and control plates were compared to estimate the degree of inhibition due to the chromogenic mixture.

\subsection{Direct detection from honey samples}

Thirty samples of honey from different origins were selected at random to test the direct isolation of Bacillus and related species on HiCrome Bacillus agar. Each sample was homogenized at $40^{\circ} \mathrm{C}$, then, $15 \mathrm{ml}$ were transferred into a screw cap centrifuge tube and mixed with an equal volume $(1,1)$ of $0.01 \mathrm{M}$ sodium phosphate buffer saline (pH 7.2). Each tube was vortex-mixed before centrifugation at $6.000 \times g$ for $45 \mathrm{~min}$ at room temperature. Most of the supernatant was discarded leaving approximately $3 \mathrm{ml}$ of fluid per tube that was then vortex-mixed for $1 \mathrm{~min}$ to resuspend the pellet and heated at $80^{\circ} \mathrm{C}$ for $10 \mathrm{~min}$ in a water bath to kill bacterial vegetative cells and yeasts and at the same time activate the germination of bacterial spores. Samples were highspeed vortex-mixed again for $2 \mathrm{~min}$, and $100 \mu \mathrm{l}$ of the sediment-fluid mixture was poured over the surface of HiCrome Bacillus agar plates and spread by using a sterile cotton swab. Plates of MYPGP were used as controls. Plates were incubated at $37{ }^{\circ} \mathrm{C}$ and examined daily and up to 10 days for bacterial growth. Distinct colonies were randomly selected from the agar plates of each sample and tested by the procedures described in Section 2.4.

\section{Results and discussion}

\subsection{Characteristics and differential properties of bacterial strains in HiCrome Bacillus}

Table 1 summarizes the growth characteristics and medium pigmentation on 197 strains belonging to different spore-forming bacteria commonly found in honey samples when growing in HiCrome Bacillus agar, together with growth characteristics and E values obtained for each tested strain. Color and appearance of colonies and media are shown in Supplementary Table 3 (Data in Brief).

Colonies of Bacillus cereus ATCC 11778 (Supplementary Fig. 1A, Data in Brief), Bacillus thuringiensis (ATCC 10792) and Bacillus mycoides (ATCC 10206) (Supplementary Table 3, Data in Brief) were flat, large and blue in color because X-glu (5-bromo-4chloro-3indolyl- $\beta$-D-glucopyranoside) present in the chromogenic mixture is cleaved by the enzyme $\beta$-glucosidase produced by these species resulting in the formation of blue colonies mainly with darker blue centers. The medium remains pinkish beige because $B$. cereus sensu lato strains do not utilize mannitol as a fermentable carbohydrate and the $\mathrm{pH}$ of the medium remains neutral. The $\mathrm{E}$ values for $B$. cereus (Supplementary Fig. 2 Data in Brief), $B$. thuringiensis, and $B$. mycoides growing on HiCrome Bacillus agar were 5, 5, and 4, respectively (Table 1). Also, 81 B. cereus, 5 B. thuringiensis, and $2 \mathrm{~B}$. mycoides strains isolated from honey samples showed the same characteristics (Table 1 and Supplementary Table 1 in Data in Brief) except one atypical strain of B. cereus (m388) that showed green colonies and turned the medium yellow (Supplementary Table 3, Data in Brief) due to utilization of peptides that release acidic compounds. B. cereus $\mathrm{m} 388$ is negative for mannitol utilization when testing by API strips or classical biochemical tests (Section 2.4) and showed the typical characteristics of $B$. cereus when growing in PEMBA (polymyxin-pyruvate-egg-yolk-mannitol agar, Holbrook and Andersson, 1980; López and Alippi, 2007). Atypical isolates of B. cereus that correlates to emetic strains connected to food-poisoning have been described by other authors (Ehling-Schulz et al., 2004; Pirttijärvi et al., 2000).

Bacillus megaterium (NRRL B-939) produced smooth, circular, glistening, mucoid and large yellow colonies turning the medium yellow due to the fermentation of mannitol that acidifies the substrate and phenol red $\mathrm{pH}$ indicator turned to yellow (Table 1, Supplementary Fig. 1B and Supplementary Table 3, Data in Brief). The same characteristics were exhibited by seven strains from honey identified as $B$. megaterium (Table 1). Interestingly, $B$. megaterium showed distinct colonies and color changes of the medium that allows confirmation of its identity without further testing.

In the case of Bacillus firmus (ATCC 8247) (Table 1 and Supplementary Table 3 (Data in Brief), Bacillus licheniformis (NRRL B-1001) (Table 1 and Supplementary Table 3 (Data in Brief), Bacillus pumilus (ATCC 7061) (Table 1, Supplementary Fig. 1I and Supplementary Table 3 in Data in Brief) and Bacillus subtilis (ATCC 6633) (Table 1, Supplementary Fig. 1E and Supplementary Table 3 in Data in Brief), bacterial colonies were light green to green due to the cleave of X-glu. Also, the medium appears yellow by mannitol utilization because the indicator phenol red turns yellow at acid $\mathrm{pH}$. According to the literature (Gordon et al., 1973; Priest et al., 1988), these species utilized mannitol as a carbon source. Similar characteristics were observed with four $B$. licheniformis (Supplementary Fig. 1H, and Supplementary Table 3, Data in Brief), and 20 B. pumilus (Supplementary Fig. 1I and Supplementary Table 3, Data in Brief) strains isolated from honey. Nevertheless, two morphological types of $B$. subtilis have been noticed, the conventional type ( 9 out of 31 ) that yielded greenish to green colonies and turned the medium yellow (Supplementary Fig. 1E and Supplementary Table 3, Data in Brief) as reported in the technical data for Bacillus subtilis subsp. spizizenii (Sigma-Aldrich, 2013) and the atypical type (22 out of 31 isolates) that showed whitish irregular, mucoid and wrinkle colonies (Supplementary Fig. 1F and Supplementary Table 3, Data in Brief) with or without detectable color changes in the background of the medium.

Bacillus badius (ATCC 14574) and Bacillus coagulans (NRRL NRS 609) produced pinkish to pink colonies and turned the medium fuchsia pink (Table 1 and Supplementary Table 3 in Data in Brief). B. badius and $B$. coagulans did not utilize or produce variable results with mannitol respectively (Gordon et al., 1973; Priest et al., 1988). Also, according to technical data of HiCrome medium, B. coagulans produced pink, small, mucoid and raised colonies and pink coloration of the medium (Sigma-Aldrich, 2013). Only B. coagulans and B. badius strains from culture collections were tested because we did not find these species in any of the honey samples examined.

Regarding Brevibacillus strains, Br. borstelensis formed pink, glistening, irregular colonies that alkalinized the medium to a magenta color (Table 1 and Supplementary Table 3 (Data in Brief), which means that Br. borstelensis neither use mannitol nor x-glu but use peptides as substrate releasing alkaline compounds. In contrast, the four strains of $B r$. laterosporus tested show green and irregular colonies coloring the medium yellowish (Table 1 and Supplementary Table 3 in Data in Brief) and Br. brevis (ATCC 8246) formed green, irregular and glistening colonies coloring the medium yellowish green (Table 1 and Supplementary Table 3 (Data in Brief).

Lysinibacillus sphaericus (ATCC 245), and also L. sphaericus $(\mathrm{n}=2)$ and $L$. fusiformis $(\mathrm{n}=1)$ isolated from honey formed pink colonies and color the medium fuchsia (Table 1, Supplementary Fig. 1G, and Supplementary Table 3 in Data in Brief). Both species have been reported as negative for mannitol fermentation (Ahmed et al., 2007; Gordon et al., 1973; Priest et al., 1988), and as observed here, these strains also alkalinized the substrate using the peptides of the medium but were not able to split X-glu. Similar characteristics of coloration in colonies and medium were observed in the case of Rummeliibacillus stabekisii mv111 (Table 1; Supplementary Table 3 and Supplementary Fig. 3, Data in Brief).

Finally, strains belonging to the genus Paenibacillus showed variable types of colonies according to the species tested. Paenibacillus alvei (NRRL B-383) and also three isolates obtained from honey, formed dark green, punctiform and raised colonies and colored the medium 
Table 1

Differential properties of Bacillus, Brevibacillus, Lysinibacillus, Paenibacillus and Rummeliibacillus species tested in HiCrome Bacillus agar.

\begin{tabular}{|c|c|c|c|c|c|}
\hline Species & $\begin{array}{l}\text { No. of strains } \\
\text { tested }\end{array}$ & Colony characteristic(s) $)_{*}$ & Medium pigmentation $_{*}$ & Growth $_{* *}$ & $\mathrm{E}_{* * *}$ \\
\hline Control & N/A & N/A & Pinkish beige [PMS 152] & N/A & N/A \\
\hline Bacillus amyloliquefaciens & 7 & Whitish, irregular, mucoid, wrinkle [PMS 468] & $\begin{array}{l}\text { Yellow [PMS 109]/Pinkish beige [PMS } \\
\text { 152] }\end{array}$ & +++ & 5 \\
\hline Bacillus badius & 1 & Pinkish, smooth, glistening, mucoid [PMS 224] & Fuchsia pink [PMS 219] & $++/+++$ & 3.8 \\
\hline Bacillus cereus & 82 & Blue, with dark blue centers, large [PMS2746] & Pinkish beige [PMS 152]/Pink [PMS 238] & +++ & 5 \\
\hline Bacillus cereus & 1 & Green [PMS 361] & Yellow [PMS 114] & +++ & 5 \\
\hline Bacillus circulans & 1 & Greenish [PMS 364] & Yellowish [PMS 390] & ++ & 4 \\
\hline Bacillus clausii & 2 & Yellowish, glistening [PMS 389] & Yellowish [PMS 389] & + & 1.6 \\
\hline Bacillus coagulans & 1 & Pink [PMS 223] & Pink [PMS 224] & $++/+++$ & 5 \\
\hline Bacillus firmus & 1 & Green, irregular, glistening [PMS 361] & Yellow [PMS 102] & ++ & 5 \\
\hline Bacillus licheniformis & 5 & Green, glistening, irregular [PMS 338] & Greenish yellow [PMS 381] & ++ & 5 \\
\hline Bacillus megaterium & 8 & $\begin{array}{l}\text { Yellow, smooth, circular, glistening, mucoid, large [PMS } \\
\text { 3945] }\end{array}$ & Yellow [PMS 604] & ++ & 4.6 \\
\hline Bacillus mycoides & 3 & Blue, large, filamentous, filliform [PMS 534] & Pink [PMS 198] & +++ & 4 \\
\hline Bacillus pumilus & 21 & Green, irregular [PMS 363] & Yellowish [PMS 383] & +++ & 5 \\
\hline Bacillus subtilis & 31 & $\begin{array}{l}\text { Whitish, irregular, mucoid, wrinkle [PMS 615] or Green } \\
\text { [PMS 346] }\end{array}$ & $\begin{array}{l}\text { Yellow [PMS 113]/Pinkish beige [PMS } \\
\text { 152] }\end{array}$ & $++/+++$ & 3 \\
\hline Bacillus thuringiensis & 6 & Light blue, large [PMS 2935] & Pinkish [PMS 673] & +++ & 5 \\
\hline Brevibacillus borstelensis & 2 & Pink, glistening, irregular [PMS 182] & Magenta [PMS 206] & $++/++$ & 3.8 \\
\hline Brevibacillus brevis & 1 & Green, irregular, glistening [PMS 360] & Yellowish green [PMS 379] & + & 4.4 \\
\hline Brevibacillus laterosporus & 4 & Green, irregular [PMS 362] & Yellowish [PMS 386] & $+/++$ & 2.2 \\
\hline Lysinibacillus fusiformis & 1 & Pink, irregular [PMS 709] & Fuchsia [PMS 231] & ++ & 3.8 \\
\hline Lysinibacillus sphaericus & 3 & Pinkish, irregular [PMS 707] & Fuchsia [PMS 232] & ++ & 1.4 \\
\hline Paenibacillus alvei & 4 & Dark green, punctiform, raise [PMS 357] & Brownish yellow [PMS 130] & + & 1 \\
\hline Paenibacillus amylolyticus & 1 & Green, punctiform [PMS 377] & Yellowish [PMS 389] & + & 5 \\
\hline Paenibacillus apiarius & 1 & Green, small [PMS 350] & Yellow [PMS 604] & $+/++$ & 4 \\
\hline Paenibacillus larvae ERIC I & 4 & Pinkish, irregular, raised [PMS 1635] & Pinkish [PMS 176] & + & 2 \\
\hline Paenibacillus larvae ERIC II & 3 & Yellowish, glistening, irregular [PMS 389] & Yellowish [PMS 379] & + & 3.8 \\
\hline Paenibacillus larvae ERIC IV & 1 & Yellowish, irregular [PMS 387] & Yellowish [PMS 388] & + & 2 \\
\hline Paenibacillus polymyxa & 1 & Green, glistening, mucoid, raised [PMS 348] & Yellowish [PMS 128] & $++/+++$ & 5 \\
\hline Rummeliibacillus stabekisii & 1 & Pink, irregular [PMS 509] & Fuchsia pink [PMS 1915] & ++ & 5 \\
\hline Total & 197 & & & & \\
\hline
\end{tabular}

N/A: not applicable.

* Pantone ${ }^{\circledR}$ Matching System (PMS) colour chart designation between brackets.

$*^{* *}+$ poor growth, ++ good growth, and +++ luxuriant growth.

*** E: ecometric value.

brownish yellow (Table 1, Supplementary Fig. 1C and Supplementary Table 3 in Data in Brief). Paenibacillus amylolyticus (NRRL B-14940), as well as Paenibacillus apiarius (ATCC 29575) and Paenibacillus polymyxa (NRRL B-510), formed green colonies that colored the medium yellow (Table 1 and Supplementary Table 3 in Data in Brief). Paenibacillus larvae isolates showed different colors of colonies and media according to the strain tested, for example, $P$. larvae belonging to genotype ERIC I (4 isolates, including the type strain ATCC 9545) showed pinkish, irregular and raised colonies and the medium remain pinkish because this group cannot utilize mannitol (Table 1 and Supplementary Table 3, Data in Brief). In contrast, $P$. larvae strains belonging to genotype ERIC II (3 isolates) formed yellowish, glistening and irregular colonies that turned the medium yellowish and P. larvae ERIC IV (ATCC 13537) formed yellowish irregular colonies on a yellowish medium (Table 1 and Supplementary Table 3, Data in Brief). It had been reported that genotypes ERIC II and IV utilized mannitol whereas genotype ERIC I did not (OIE, 2016). Genotype ERIC I includes the former P. larvae subsp. larvae and genotypes ERIC II and IV the previous P. larvae subsp. pulvifaciens, today reclassified as $P$. larvae without subspecies differentiation (Genersch et al., 2006; OIE, 2016). P. larvae is the causal agent of American Foulbrood of honeybees, the most devastating bacterial disease affecting honeybee brood worldwide (Genersch, 2010) and bacterial spores present in honey can transmit the disease between colonies and apiaries, remaining infective for many years. Colony counts are highly variable from null or few numbers of spores to thousands per gram of honey (Alippi et al., 2004). When testing P. larvae vegetative cells of different isolates $(n=8)$ on HiCrome Bacillus agar, bacterial growth was rather poor with Ecometric code values ranging from 2 to 3.8 (Table 1 and Supplementary Table 3, Data in Brief) in comparison with values of 5 obtained when using MYPGP control medium (Supplementary Table 3, Data in Brief). Spores were excluded because $24 \mathrm{~h}$ cultures were used and specific media and at least 3 days are required to sporulate. Routine bacteriological culture media do not support the growth of $P$. larvae, and also there are not reliable methods for making plate counts by using spores because fewer than $10 \%$ of spores produce visible growth on the presently available media for cultivation (Dingman and Stahly, 1983; OIE, 2016). Further studies are needed to evaluate if the supplementation of HiCrome with other components could allow the germination of $P$. larvae spores for its application in the analysis of honey.

We tested 176 strains belonging to 24 species of different genera that had been reported in honey, including 21 strains from Culture Collections and determined different types of colonies and colors and also different colors of the medium, i.e., yellowish, pinkish, green to greenish or whitish colonies with yellow or yellowish medium, pink or fuchsia pink medium. Results obtained when testing the strains isolated from honey, and apiarian sources (Supplementary Tables 1 and 3, Data in Brief) gave consistent results with those obtained with strains from Culture Collections.

Results obtained here on colony appearance on HiCrome Bacillus agar are in agreement on those reported for B. cereus sensu lato, $B$. megaterium, $B$. pumilus and $B$. coagulans in the technical information from Sigma-Aldrich (2013) and also on those reported by (Nemecková et al., 2011) for B. cereus and for B. cereus and B. licheniformis strains 


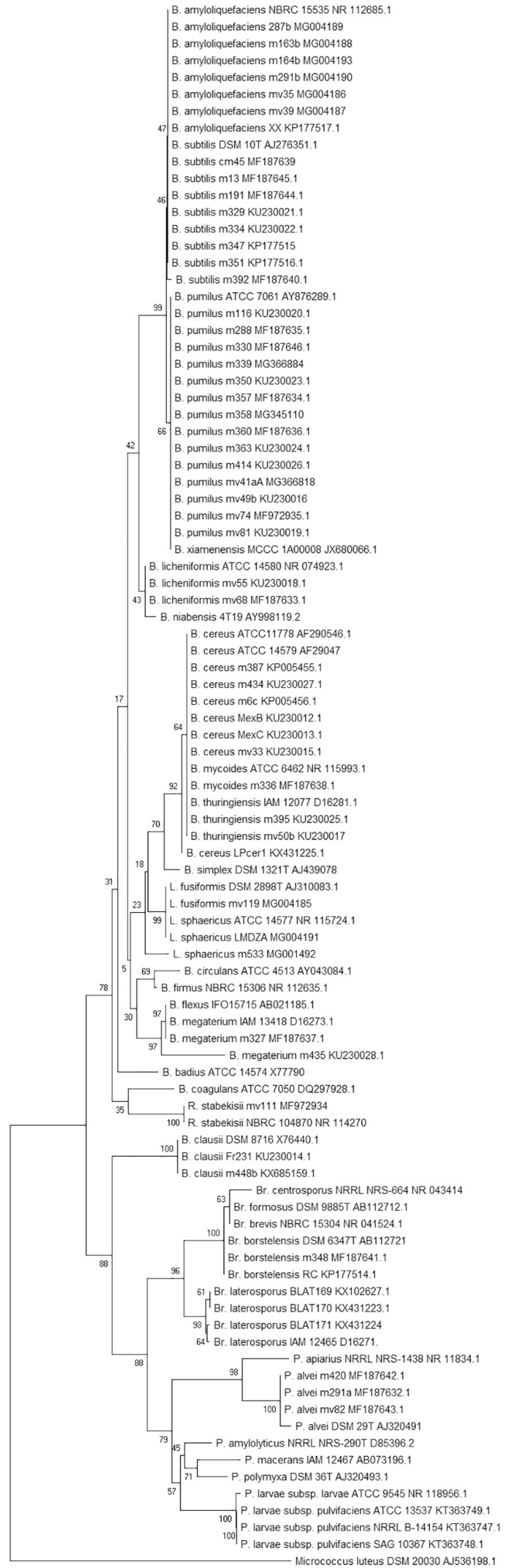

Fig. 1A. Neighbor-Joining (NJ) phylogenetic tree based on partial 16S rRNA data showing the phylogenetic relationships of Bacillus, Brevibacillus, Lysinibacillus, Paenibacillus, and Rummeliibacillus isolates commonly found in honey, rooted using Micrococcus luteus DSM $20030^{\mathrm{T}}$ as an outgroup. Bootstrap values (expressed as percentages of 1000 replicates) are shown next to the branches. The evolutionary distances were computed using the p-distance method and are in the units of the number of base differences per site. The analysis involved 93 nucleotide sequences. All positions containing gaps and missing data were eliminated. There were a total of 422 positions in the final dataset. Evolutionary analyses were conducted in MEGA X. Bar, 0.020 substitutions per site.

isolated from raw milk.. In the case of $B$. subilis, technical information from the suppliers (Sigma-Aldrich, 2013) describes B. subtilis ATCC 6633 as producing light green to green colonies, but this particular strain from ATCC belongs to Bacillus subtilis subsp. spizizenii, so data are not comparable since we did not test this subspecies. As far as we know, this is the first description of types of colonies and colors produced by B. amyloliquefaciens, B. badius, B. circulans, B. clausii, B. firmus, Br. borstelensis, Br. brevis, Br. laterosporus, L. fusiformis, L. sphaericus, $P$. alvei, $P$. amylolyticus, $P$. apiarius, $P$. larvae, $P$. polymyxa and $R$. stabekisii when growing on HiCrome Bacillus agar or any chromogenic medium.

\subsection{Identification of bacterial isolates and phylogenetic analysis}

Based on both, Blast and EZBiocloud searches of the partial $16 \mathrm{~S}$ rRNA gene sequences, Genbank accession numbers were assigned to 56 isolates (Supplementary Table 1, Data in Brief). All searches correlated with the presumptive identification obtained from the combination of selected biochemical tests and the characteristics observed in the chromogenic medium. Also, information from the phylogenetic tree reconstruction employing both maximum-likelihood (Fig. 1B) and neighbor-joining (Fig. 1A) methods revealed that the branches are conserved in the two trees.

Identification of isolates from honey by using a combination of selected biochemical and morphological tests and HiCrome Bacillus agar.

A flowchart was prepared by a combination of colony and media characteristics in HiCrome Bacillus agar and a set of selected biochemical and morphological tests that are used routinely in Microbiological laboratories (Supplementary Fig. 4 in Data in Brief). Following the simple steps of the chart, we were able to successfully identify the most common aerobic spore-forming species associated with honey and other apiarian sources. The proposed key is practical and convenient to use since it permits species identification by a few simple tests. A simplified flowchart is shown in Supplementary Fig. 5 in Data in Brief that allows differentiating typical strains of aerobic sporeforming species reported in honey. In some cases, for additional confirmation, other tests may be needed.

It is important to notice that, in the case of some species, i.e., $B$. clausii, L. sphaericus, P. larvae, P. alvei, and P. amylolyticus poor growth and low ecometric values were observed in HiCrome medium in comparison with controls (Table 1 and Supplementary Table 3 in Data in Brief), mainly if the inoculum source is a spore suspension (Data not shown). Further studies are needed to evaluate if the chromogenic mixture inhibits the germination of bacterial spores of particular species.

\section{Conclusion}

In conclusion, HiCrome Bacillus agar in conjunction with simple biochemical tests was highly useful for the presumptive identification of most Bacillus, Brevibacillus, Lysinibacillus and Paenibacillus species commonly found in honey samples facilitating isolation from polymicrobial honey. 


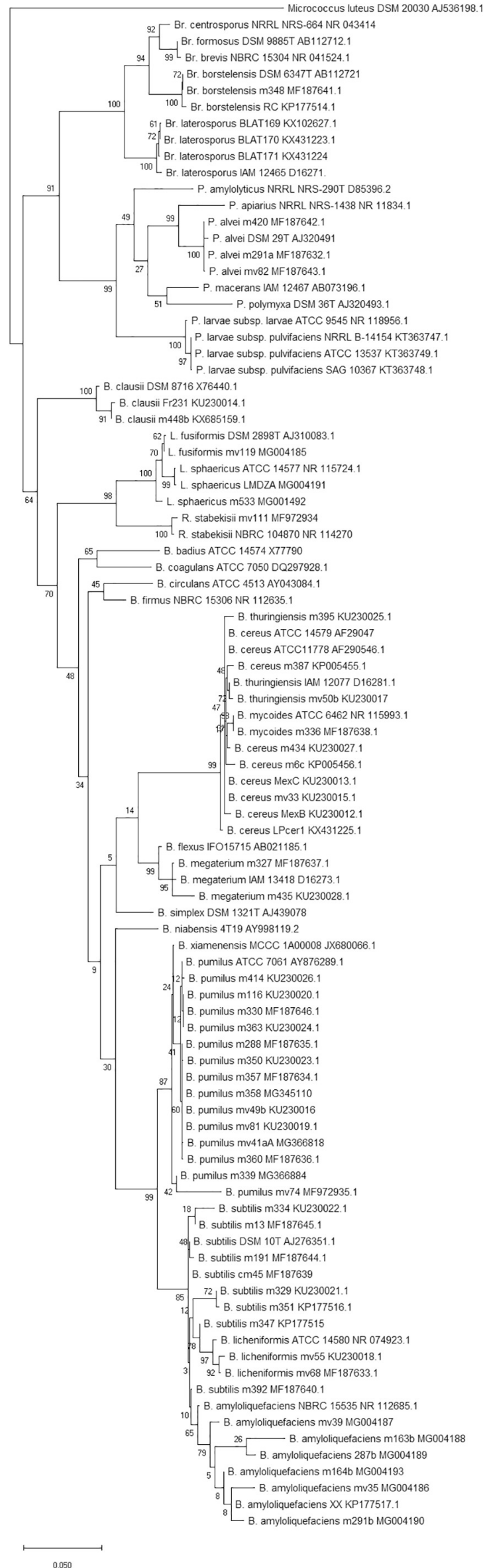

Fig. 1B. Molecular Phylogenetic analysis by Maximum Likelihood (ML) method based on partial 16S rRNA gene sequences of Bacillus, Brevibacillus, Lysinibacillus, Paenibacillus, and Rummeliibacillus isolates commonly found in honey. The evolutionary history was inferred by using the Maximum Likelihood method based on the Jukes-Cantor model. The tree with the highest log likelihood $(-14,759.53)$ is shown. The tree is drawn to scale, with branch lengths measured in the number of substitutions per site. The analysis involved 93 nucleotide sequences. All positions containing gaps and missing data were eliminated. There were a total of 1895 positions in the final dataset. Evolutionary analyses were conducted in MEGA X. Micrococcus luteus DSM $20030^{\mathrm{T}}$ [AJ536198.1] was used as an outgroup in the tree.

\section{Declaration of Competing Interest}

The authors declare that no conflict of interest exists.

\section{Acknowledgements}

This work was financially supported by the Agencia Nacional de Promoción Científica y Tecnológica (ANPCyT) [PICT 2012/0189 and PICT 2017/2014] and the Comisión de Investigaciones Científicas de la Provincia de Buenos Aires (CICBA) [Grants 1194/14 and 274/16]. AMA is a member of the Scientific Research Career of CICBA and EA is a doctoral fellow from CONICET (CCT-La Plata).

We thank Dr. Ivo Siegrist for his helpful advice on HiCrome Bacillus agar.

\section{Appendix A. Supplementary data}

Supplementary data to this article can be found online at https:// doi.org/10.1016/j.ijfoodmicro.2019.108245.

\section{References}

Aguilera-Arreola, M.G., Portillo-Muñoz, M.I., Rodríguez-Martínez, C., Castro-Escarpulli, G., 2012. Usefulness of Chromogenic CromoCen ${ }^{\circledR}$ AGN agar medium for the identification of the genus Aeromonas: assessment of fecal samples. J. Microbiol. Methods 90, 100-104.

Ahmed, I., Yokota, A., Yamazoe, A., Fujiwara, T., 2007. Proposal of Lysinibacillus boronitolerans gen. nov. sp. nov., and transfer of Bacillus fusiformis to Lysinibacillus fusiformis comb. nov. and Bacillus sphaericus to Lysinibacillus sphaericus comb. nov. Int. J. Syst. Evol. Microbiol. 57, 1117-1125.

Alippi, A.M., 1995. Detection of Bacillus larvae spores in Argentinian honeys by using a semi-selective medium. Microbiología (Madrid) 11, 343-350.

Alippi, A.M., Reynaldi, F.J., López, A.C., De Giusti, M.R., Aguilar, O.M., 2004. Molecular epidemiology of Paenibacillus larvae larvae and incidence of American Foulbrood in Argentinean honeys from Buenos Aires Province. J. Apic. Res. 43, 135-143.

Applied Biosystems, 2009. Chapter 5: Purification of extension products. In: Applied Biosystems Chemistry Guide (Ed.), DNA Sequencing by Capillary Electrophoresis Chemistry Guide, Foster City, USA, pp. 85-122. https://www.thermofisher.com/ar/ es/home/life-science/sequencing/sequencing-applications.html\#seq.

Dingman, D.W., Stahly, D.P., 1983. Medium promoting sporulation of Bacillus larvae and metabolism of medium components. Appl. Environ. Microbiol. 46, 860-869.

Edgar, R.C., 2004. MUSCLE: multiple sequence alignment with high accuracy and high throughput. Nucleic Acids Res. 32, 1792-1797.

Ehling-Schulz, M., Fricker, M., Scherer, S., 2004. Review. Bacillus cereus, the causative agent of an emetic type of food-borne illness. Mol. Nutr. Food Res. 48, 479-487.

Evans, J.D., Armstrong, T.-N., 2006. Antagonistic interactions between honey bee bac terial symbionts and implications for disease. BMC Ecol. 6, 4. https://doi.org/10. 1186/1472-6785-6-4.

Felsenstein, J., 1985. Confidence limits on phylogenies: an approach using the bootstrap. Evolution 39, 783-791.

Fricker, M., Reissbrodt, R., Ehling-Schultz, M., 2008. Evaluation of standard and new chromogenic selective plating media for isolation and identification of Bacillus cereus. Int. J. Food Microbiol. 121, 27-34.

Genersch, E., 2010. American Foulbrood in honeybees and its causative agent, Paenibacillus larvae. J. Invertebr. Pathol. 103, S10-S19. https://doi.org/10.1016/j. jip. 2009.06.015.

Genersch, E., Forsgren, E., Pentikäinen, J., Ashiralieva, A., Rauch, S., Kilwinski, J., Fries, I., 2006. Reclassification of Paenibacillus larvae subsp. pulvifaciens and Paenibacillus larvae subsp. larvae as Paenibacillus larvae without subspecies differentiation. Int. J. Syst. Evol. Microbiol. 56, 501-511.

Gilliam, M., 1979. Microbiology of pollen and bee bread: the genus Bacillus. Apidologie 10, 269-274.

Gordon, R.E., Haynes, W.C., Pang, C.H.-N., 1973. The Genus Bacillus: Agriculture 
Handbook No. 427. Agricultural Research Service, USDA, Washington, D.C.

Holbrook, R., Andersson, J.M., 1980. An improved selective and diagnostic medium for the isolation and enumeration of Bacillus cereus in foods. Can. J. Microbiol. 26, 753-759.

Iurlina, M.O., Fritz, R., 2005. Characterization of microorganisms in Argentinean honeys from different sources. Int. J. Food Microbiol. 105, 297-304.

Jeyaprakash, A., Hoy, M.A., Allsopp, M.H., 2003. Bacterial diversity in worker adults of Apis mellifera capensis and Apis mellifera scutellata (Insecta: Hymenoptera) assessed using 16S rRNA sequences. J. Invertebr. Pathol. 84, 96-103.

Juergensmeyer, M.A., Gingras, B.A., Restaino, L., Frampton, E.W., 2006. A selective chromogenic agar that distinguishes Bacillus anthracis from Bacillus cereus and Bacillus thuringiensis. J. Food Prot. 69, 2002-2006.

Kornacki, J.L., Gurtler, J.B., Yan, Z., Cooper, C.H., 2003. Evaluation of several mod ifications of the ecometric technique for assessment of media performance. J. Food Prot. 66, 1727-1732.

Kumar, S., Stecher, G., Li, M., Knyaz, C., Tamura, K., 2018. MEGA X: molecular evolutionary genetics analysis across computing platforms. Mol. Biol. Evol. 35, 1547-1549.

Logan, N.A., Berge, O., Bishop, A.H., Busse, H.J., De Vos, P., Fritze, D., Heyndrickx, M., Kampfer, P., Rabinovitch, L., Salkinoja-Salonen, M.S., Seldin, L., Ventosa, A., 2009. Proposed minimal standards for describing new taxa of aerobic, endospore-forming bacteria. Int. J. Syst. Bacteriol. 59, 2114-2121.

López, A.C., Alippi, A.M., 2007. Phenotypic and genotypic diversity of Bacillus cereus isolates recovered from honey. Int. J. Food Microbiol. 117, 175-184.

Nei, M., Kumar, S., 2000. Molecular Evolution and Phylogenetics. Oxford University Press, New York.

Nemecková, I., Solichová, K., Roubal, P., Uhrová, B., Sviráková, E., 2011. Methods for detection of Bacillus sp., B. cereus, and B. licheniformis in raw milk. Czech J. Food Sci. 29, S55-S60.

OIE, 2016. Chapter 2.2.2. American foulbrood of honey bees. (infection of honey bees with Paenibacillus larvae). In: OIE. Terrestrial Animal Health Code, OIE, France, 17 pp.. http://www.oie.int/fileadmin/Home/eng/Health_standards/tahm/2.02.02 AMERICAN_FOULBROOD.pdf.

Parry, J.M., Turnbull, P.C.B., Gibson, J.R., 1983. A Colour Atlas of Bacillus Species. Wolfe Medical Publications Ltd., Ipswich, England.

Perry, J.D., Freydiere, A.M., 2006. Review article: the application of chromogenic media in clinical microbiology. J. Appl. Microbiol. 103, 2046-2055.

Piccini, C., Antúnez, K., Zunino, P., 2004. An approach to the characterization of the honey bee hive bacterial flora. J. Apic. Res. 43, 101-104.

Pirttijärvi, T.S.M., Andersson, M.A., Salkinoja-Salonen, M.S., 2000. Properties of Bacillus cereus and other bacilli contaminating biomaterial-based industrial processes. Int. J. Food Microbiol. 60, 231-239.

Priest, F.G., Goodfellow, M., Todd, C., 1988. A numerical classification of the genus Bacillus. J. Gen. Microbiol. 143, 1847-1882.

Siegrist, J., 2016. Update to chromogenic media. In: Microbiology Focus. vol. 8.1 SigmaAldrich.

Sigma-Aldrich, 2013. Product Information. 92325 Bacillus ChromoSelect Agar (Bacillus cereus ChromoSelect Agar - HiCrome ${ }^{\mathrm{TM}}$ Bacillus Agar - Bacillus cereus HiCrome ${ }^{\mathrm{TM}}$ Agar). Sigma-Aldrich.com.

Silva, M.S., Rabadzhiev, Y., Eller, M.R., Iliev, I., Ivanova, I., Santana, W.C., 2017. Chapter 11: Microorganisms in honey. In: de Toledo, V.A. (Ed.), Honey Analysis, pp. 233-234. https://doi.org/10.5772/63259.

Sinacori, M., Francesca, N., Alfonzo, A., Cruciata, M., Sannino, C., Settanni, L., Moschetti, G., 2014. Cultivable microorganisms associated with honeys of different geographical and botanical origin. Food Microbiol. 38, 284-294.

Snowdon, J.A., Cliver, D.O., 1996. Microorganisms in honey. Int. J. Food Microbiol. 31, $1-26$.

Tewari, A., Singh, S.P., Singh, R., Kumar, D., 2013. Comparison of a new chromogenic medium with standard media for isolation and identification of Bacillus cereus. Eurasian J. Vet. Sci. 29, 39-42.

Wen, Y., Wang, L., Yue, J., Zhang, J., Su, L., Zhang, X., Zhou, J., Li, Y., 2017. The microbial community dynamics during the vitex honey ripening process in the honeycomb. Front. Microbiol. 8, 1649. https://doi.org/10.3389/fmicb.2017.01649.

Yoon, S.-H., Ha, S.-M., Kwon, S., Lim, J., Kim, Y., Seo, H., Chun, J., 2017. Introducing EzBioCloud: a taxonomically united database of 16S rRNA gene sequences and whole-genome assemblies. Int. J. Syst. Evol. Microbiol. 67, 1613-1617.

Yu, J., Zhou, X.F., Yang, S.J., Liu, W.H., Hu, X.F., 2013. Design and application of specific 16S rDNA-targeted primers for assessing endophytic diversity in Dendrobium officinale using nested PCR-DGGE. Appl. Microbiol. Biotechnol. 97, 9825-9836. https://doi. org/10.1007/s00253-013-5294-y. 